\title{
Infinite Limits of Copying Models of the Web Graph
}

\author{
Anthony Bonato and Jeanette Janssen
}

Abstract. Several stochastic models were proposed recently to model the dynamic evolution of the web graph. We study the infinite limits of the stochastic processes proposed to model the web graph when time goes to infinity. We prove that deterministic variations of the so-called copying model can lead to several nonisomorphic limits. Some models converge to the infinite random graph $R$, while the convergence of other models is sensitive to initial conditions or minor changes in the rules of the model. We explain how limits of the copying model of the web graph share several properties with $R$ that seem to reflect known properties of the web graph.

\section{Introduction}

The web may be viewed as a directed graph with nodes representing the static HTML web pages, and directed edges representing the links between web pages. This graph is commonly referred to as the web graph; it is an example of a massive network, with several billion nodes. Several interesting properties were observed in the web graph: in particular, the in- and out-degrees seem to satisfy a power law degree distribution, the web graph is small world, which means that it has high clustering and low diameter, and it is locally dense while globally sparse. (See [Kumar et al. 00b] for a survey of properties of the web graph.) Another interesting property of the web graph is that it is evolutionary: nodes appear and disappear with time.

Owing to its massive and dynamic nature, several authors have suggested statistical models which capture certain properties of the web graph. These models

(C) A K Peters, Ltd.

I542-795I/04 $\$ 0.50$ per page 
are loosely based on classical random graphs, first introduced by Erdős and Rényi [Erdős and Rényi 60]. If $n$ is a positive integer, and $0<p<1$ is a fixed real number, then a random graph $G(n, p)$ has $n$ nodes, and there is an edge between two nodes with probability $p$. The graphs $G(n, p)$ have several drawbacks as models of the web graph. For example, the degree distribution of random graphs is binomial, rather than satisfying a power law; further, the number of nodes is static. These drawbacks may be overcome by making the model dynamic, and by assigning different probabilities to various nodes. Two models that take these approaches are the preferential attachment model of Barabási and Albert [Barabàsi and Albert 99] and the evolving copying model of Kumar et al. [Kumar et al. 00a]. In the preferential attachment model, we start with a small base graph. At each time step, we create a new node, say $u$, and draw its edges according to a predetermined distribution. In particular, node $u$ is joined to an existing node $v$ with probability proportional to $\operatorname{deg}(v)$. In the evolving copying model, we start with a small base graph. At each time step, we create a new node, $u$. Choose an existing node $v$ uniformly at random (u.a.r.). For each edge $v w$, with probability $p$, add the edge $u w$. Hence, the neighbourhood of the new node $u$ will be a subset of the neighbourhood of the existing node $v$. A slight variation is the evolving copying model with error, where with probability $1-p$, an edge is added between $u$ and an existing node chosen u.a.r. (In some variants of the model, an additional number of random neighbours are added to the new node $u$ instead; see [Adler and Mitzenmacher 01]. We note also that there are both directed and undirected versions of this and other web graph models.)

The first analysis of the long-term behaviour of these models has been made, for example, by Aiello, Chung, and Lu [Aiello et al. 00, Aiello et al. 01]; Bollobás et al. [Bollobás et al. 01]; Cooper and Frieze [Cooper and Frieze 01]; and Kumar et al. [Kumar et al. 00a]. Power law degree distributions were proven to exist in both the preferential attachment [Barabàsi and Albert 99] and evolving copying models [Kumar et al. 00a]. Many bipartite cliques were shown to exist in evolving copying models [Kumar et al. 00a], mirroring the abundance of so-called "cyber-communities" measured by bipartite cliques in the web graph (as reported in [Kumar et al. 99]).

Our motivating question is: what are the resulting graphs like if we allow these stochastic processes to continue indefinitely? We attempt to answer this question in the case of the copying model of the web graph. The resulting graphs are infinite, and are limits (that is, unions of chains) of finite web graph models. On the surface, the study of infinite graphs may appear to have no connection with the study of a finite experimental graph such as the web graph. Indeed, we draw no explicit connections between many of the properties observed in the infinite limits and the finite graphs. However, limits of web graph models 
have certain properties (such as fractal or self-similarity properties) which are implicitly linked with known data on the web graph obtained by various web crawls. (See Theorems 4.1 and 4.6.)

Throughout this paper, we will consider the simple, undirected version of the web graph. (The reason for this is that the structural results we present are proved only for undirected graphs; we consider the directed case as the next step in our study.) If we fix any $p \in(0,1)$ and consider limits of the $G(n, p)$ graphs, then the resulting graph will almost surely be isomorphic to the infinite random graph, written $R$. The graph $R$ is the unique (up to isomorphism) countable graph satisfying the following existentially closed (e.c.) adjacency property.

e.c. property. A graph $G$ is e.c. if for each pair of finite disjoint subsets $X$ and $Y$ of nodes of $G$, there exists a node $z_{X, Y} \in V(G) \backslash(X \cup Y)$ that is joined to each node of $X$ and to no node of $Y$.

For more on $R$, the reader is directed to the excellent survey [Cameron $97 \mathrm{~b}$ ]. The graph $R$ may be viewed as the limit of an evolutionary process. For this, let $R_{0}$ be a single node; assume that $R_{n}$ is defined and contains $R_{0}$. Enumerate all of the finite subsets of nodes of $R_{n}$, and extend each of these subsets, in all possible ways, by new nodes not in $R_{n}$. The resulting graph we call $R_{n+1}$, and the union of the chain $\left(R_{n}: n \in \omega\right)$ is an e.c. graph that is isomorphic to $R$. The preceding construction of $R$ serves as a template for what follows, where we will consider infinite graphs grown by certain evolutionary processes. Our results show that graphs grown in this way have many properties in common with $R$, although they are not usually isomorphic to $R$. See Sections 3 and 4 .

\section{Adjacency Properties and Limits}

All the graphs we consider are undirected, simple, and have a countable number of nodes. We use the notation $\omega$ for the set of natural numbers considered as an ordinal, and $\aleph_{0}$ is the cardinality of $\omega$. The cardinality of the real numbers is written $2^{\aleph_{0}}$. If $S \subseteq V(G)$, then $G \uparrow S$ is the subgraph induced by $S$. If $G$ is an induced subgraph of $H$, then we write $G \leq H$. The graph $G \uplus H$ is the disjoint union of $G$ and $H$. If $y$ is a node of $G$, then $N(y)=\{z: y z \in E(G)\}$ is the neighbour set of $y$ in $G$. The closed neighbour set of $y$, written $N[y]$, is the set $N(y) \cup\{y\}$. If $x$ is a node of $G$, then the graph $G-x$ is the graph $G \uparrow(V(G) \backslash\{x\})$. If $S \subseteq V(G)$, then $G-S$ is defined similarly. A node is isolated if it has no neighbours, and it is universal if it joined to all nodes except itself. If two graphs $G$ and $H$ are isomorphic, then we write $G \cong H$.

To study the limits of web graph models, we consider graphs satisfying various deterministic adjacency properties that are more general than the e.c. property 
described in Section 1. We note that determinism has been used by other authors in the study of the web graph; for instance, see [Barabàsi et al. 01] for a study of deterministic scale-free networks. Let $X$ and $Y$ be disjoint finite sets of nodes in a graph $G$. We say that the node $z_{X, Y} \in V(G) \backslash(X \cup Y)$ is correctly joined to $X$ and $Y$, if $z_{X, Y}$ is joined to each node of $X$ and no node of $Y$.

Property (A). A graph $G$ has property (A) if for each node $y$ of $G$, for each finite $X \subseteq N[y]$, and each finite $Y \subseteq V(G) \backslash X$, there exists a node $z_{X, Y} \neq y$ which is correctly joined to $X$ and $Y$.

Property (B). Property (B) is defined similarly to Property (A), except that $N[y]$ is replaced by $N(y)$.

For a fixed $n \in \omega$, Properties $(\mathrm{A}, n)$ and $(\mathrm{B}, n)$ are defined analogously to (A) and (B), respectively, but the node $z$ may be joined to at most $n$ other nodes. More precisely,

Property (A, $n)$. A graph $G$ has property $(\mathrm{A}, n)$ for some $n \in \omega$ if for each node $y$ of $G$, for each finite $X \subseteq N[y]$, for each finite $Y \subseteq V(G) \backslash X$, and for each set $U \subseteq V(G) \backslash(N[y] \cup Y)$ with cardinality at most $n$, there is a node $z_{X, Y, U} \neq y$ correctly joined to $X \cup U$ and $Y$.

Property (B, $n)$. Again, Property $(\mathrm{B}, n)$ is defined similarly to Property $(\mathrm{A}, n)$, except that $N[y]$ is replaced by $N(y)$.

Note that Property $(\mathrm{A})$ is just $(\mathrm{A}, 0)$, and $(\mathrm{B})$ is just $(\mathrm{B}, 0)$. We sometimes say that a graph with property $(\mathcal{P})$, where $\mathcal{P}$ is one of $\mathrm{A}$ or $\mathrm{B}$, is a $(\mathcal{P})$ graph.

The adjacency properties (A) and (B) are inspired by the evolving copying model of the web graph, while Properties $(\mathrm{A}, n)$ and $(\mathrm{B}, n)$ are inspired by the evolving copying model with error. Such models were first introduced by Kumar et al. [Kumar et al. 00a]. The idea is that as time goes to infinity, any extension that is made with positive probability is almost surely true in the limit. To give a more precise connection between the new adjacency properties above and limits of the copying web graph models, we consider the following model of [Adler and Mitzenmacher 01] which is a small variation on the Kumar et al. model.

For the copying model, the parameters are a fixed real number $p(0<p<1)$, and a nonnegative integer $n$.

1. At $t=0$, set $G=G_{0}$, where $G_{0}$ is a fixed finite graph.

2. For a fixed $t>0$, assume $G_{t}$ has been defined. To form $G_{t+1}$, add a new node $u$ to $G_{t}$, and choose its neighbours as follows:

(a) Choose a node $v$ of $G_{t}$ u.a.r. 
(b) For each neighbour $w$ of $v$ (independently), add an edge $u w$ to $E\left(G_{t+1}\right)$ with probability $p$.

(c) Choose a set $S$ of size $n$ from $V\left(G_{t+1}\right) \backslash(N[v] \cup\{u\})$ u.a.r. Add edges $\{u x: x \in S\}$ to $E\left(G_{t+1}\right)$.

If $\left(G_{t}: t \in \omega\right)$ is a sequence of graphs with $G_{t} \leq G_{t+1}$, then define

$$
\lim _{t \rightarrow \infty} G_{t}=\bigcup_{n \in \omega} G_{t}
$$

we call $\lim _{t \rightarrow \infty} G_{t}$ the limit of the sequence $\left(G_{t}: t \in \omega\right)$. We say that $\left(G_{t}: t \in \omega\right)$ is a chain of graphs. We now show that graphs that are limits of copying models with no error (that is, with $n=0$ ) almost surely satisfy Property (B). A more detailed analysis of the infinite limits of various web graph models (including the copying models with error) will be developed in future work.

Theorem 2.I. With probability 1 , a limit $G=\lim _{t \rightarrow \infty} G_{t}$ of copying model graphs with $n=0$ has Property (B).

Proof. Since a countable union of measure 0 subsets has measure 0 , it suffices to show that for a fixed $y \in V(G)$, a finite $X \subseteq N(y)$, and a finite $Y \subseteq V(G) \backslash X$, the probability that there is no vertex correctly joined to $X$ and $Y$ is 0 (since there are only countably many choices for $y, X$, and $Y$ in $G)$.

Let $m$ be the least integer such that $y, X$, and $Y$ are in $V\left(G_{m}\right)$. Let $\left|V\left(G_{m}\right)\right|=$ $m^{\prime},|X|=i$, and $|Y \cap N(y)|=j$. If $t \geq 1$, let $u_{m+t}$ be the vertex that was added to $G_{m+t-1}$ to form $G_{m+t}$. The probability that $y$ is chosen as the copying node in $G_{m+t}$ equals $\frac{1}{m^{\prime}+t}$. Given that $y$ is the copying node, $u_{m+r}$ is joined to all of $X$ and none of $Y$ with probability $p^{i}(1-p)^{j}$. Then the probability that no vertex of $G_{m+t}$ is correctly joined to $X$ and $Y$ is at most

$$
f(t)=\prod_{k=1}^{t}\left(1-\left(\frac{1}{m^{\prime}+k}\right) p^{i}(1-p)^{j}\right) .
$$

It is not hard to see that $\lim _{t \rightarrow \infty} f(t)=0$. (For example, first take logarithms, then use the fact that $\ln (1+x) \approx x$ for small values of $x$.)

We highlight that the proof of Theorem 2.1 is similar to the original proof of Erdős and Rényi that with probability 1 a random countably infinite graph is e.c. See [Cameron 97b, Erdős and Rényi 63].

Is there anything that can be said about the structure of graphs with these adjacency properties? How do these graphs compare and contrast with $R$, and with the actual web graph? In this section and the next, we will attempt to 
answer these questions. The web graph and graphs constructed by the copying model are of course finite. Therefore, although our results are suggestive, we add the disclaimer that we cannot draw direct comparisons between observed properties of the web graph and properties of our limits.

A first observation about our new adjacency properties is that we have the following chain of logical implications (for all integers $n \geq 1$ ),

$$
\text { e.c. } \Rightarrow(\mathrm{A}, n) \Rightarrow(\mathrm{B}, n) \Rightarrow(\mathrm{A}) \Rightarrow(\mathrm{B}) \text {. }
$$

Our second theorem gives insight into the structure of graphs with (A). A graph is $\aleph_{0}$-universal if it embeds all countable graphs as induced subgraphs. For example, it well-known that $R$ is $\aleph_{0}$-universal; see [Cameron 97b], for example. A graph $G$ is locally $H$ if for all $x \in V(G), G \uparrow N(x) \cong H$.

Theorem 2.2. A graph $G$ satisfying Property (A) is locally $R$. In particular, $G$ is $\aleph_{0}$-universal.

Proof. Fix $y \in V(G)$. By remarks in Section 1, it is enough to show that $N=G$ 个 $N(y)$ is e.c. For this, fix $X$ and $Y$, disjoint subsets of $V(N)$. By Property (A) there is a node $z_{X, Y}$ of $G$ that is joined to each node of $\{y\} \cup X$, but is not joined nor equal to any of the nodes in $Y$. Then $z_{X, Y}$ is a node of $N$, and therefore, $N$ satisfies the e.c. property.

In the next theorem, we see that if $n \geq 1$, then the adjacency properties (A, $n)$ and $(\mathrm{B}, n)$ are in fact equivalent to the e.c. property. We find this surprising, since then adding a single extra "random link" gives a deterministic conclusion. As we will see in Theorem 3.4, however, there are uncountably many nonisomorphic countable graphs with Properties (A) or (B). Thus, Theorems 2.3 and 3.4 seem to contrast the evolving copying and evolving copying with error models.

Theorem 2.3. Fix $n>0$ an integer. If $G$ has Property $(B, n)$, then $G$ is isomorphic to $R$.

Proof. To show that $G$ is isomorphic to $R$, we need only show that $G$ is e.c.: for all finite disjoint subsets $C$ and $D$ of nodes of $G$, there is a node $z$ of $V(G) \backslash(C \cup D)$ that is joined to each node of $C$ and to no node of $D$.

As $C$ is finite, we may write $C$ as $W_{1} \cup \ldots \cup W_{r}$, where the $W_{i}$ are pairwise disjoint and have cardinality at most $n$. Choose a node $y$ not in $C \cup D$. Let $X=N(y) \cap W_{1}, Y=D$, and let $U=W_{1} \backslash N(y)$. By Property (B, $\left.n\right)$, there is a node $x_{1} \notin X \cup Y$ such that $W_{1} \subseteq N\left(x_{1}\right)$. By Property $(\mathrm{B}, n)$ with $X=W_{1}$, 
$Y$ empty, and $U=W_{2}$, there is a node $x_{2}$ not in $X \cup Y$ that is joined to all of $W_{1} \cup W_{2}$. Proceeding inductively, we can find a node $x_{r}$ that is joined to all of the nodes in $C$. As then $C \subseteq N\left(x_{r}\right)$, by a final application of $(\mathrm{B}, n)$, there is a node $z$ correctly joined to $C$ and $D$.

Because of Theorem 2.3, we will restrict our attention to graphs satisfying Properties $(\mathrm{A})=(\mathrm{A}, 0)$ and $(\mathrm{B})=(\mathrm{B}, 0)$ for the rest of the paper. A graph $G$ has an (A)-constructing sequence if there is a chain of graphs $\left(G_{n}: n \in \omega\right)$ such that $\lim _{n \rightarrow \infty} G_{n}=G$, which has the following properties. (A (B)-constructing sequence is defined analogously, using $N(y)$ rather than $N[y]$.)

1. $G_{0}$ is a finite graph.

2. For each integer $n>1, G_{n+1}$ is obtained from $G_{n}$ by one application of process (P1) followed by a finite number (possibly zero) applications of process (P2), where (P1) and (P2) are defined as follows:

(P1) For each node $y$ of $G_{n}$, and for each finite $X \subseteq N[y]$, a new node $z_{X} \notin V\left(G_{n}\right)$ is added whose neighbours in $V\left(G_{n}\right)$ are exactly the nodes of $X$. We say that $z_{X}$ extends $X$. We also extend $X=\emptyset$ by a new node that is not joined to any node of $G_{n}$. Note that for distinct $X$, the new nodes $z_{X}$ are distinct. We say that all subsets of closed neighbour sets in $V\left(G_{n}\right)$ are extended in all ways.

(P2) For a finite fixed $X \subseteq V\left(G_{n}\right)$, a new node $z_{X} \notin V\left(G_{n}\right)$ is added whose neighbours in $V\left(G_{n}\right)$ are exactly the nodes of $X$.

The graph $G$ is then $\lim _{n \rightarrow \infty} G_{n}$. We refer to the graphs $G_{n}$ as time-steps in the evolution of $G$. If (P2) is never used at any time-step, then we say that the corresponding construction sequence is pure; otherwise, the constructing sequence is mixed. A graph $G$ is pure if it has a pure constructing sequence. Otherwise, we say that $G$ is mixed. In a mixed constructing sequence, the nodes added in (P2) are called extra nodes. We note that $R$ has both an (A)- and (B)-constructing sequence where (P2) is used at each time-step. (We leave the details as an exercise.) We emphasize that (A)- and (B)-constructing sequences are technical tools that have no direct relationship with the copying process sequences used to form the infinite limit.

We note that a graph $G$ formed by an (A)-constructing sequence has Property (A). The converse also holds.

Lemma 2.4. Let $G$ be a graph with $V(G)=\omega$, and fix a finite induced subgraph $H$. If $\mathcal{P}$ is $A$ or $B$, and if $G$ has property $(\mathcal{P})$, then $G$ has an $(\mathcal{P})$-constructing sequence $\left(G_{n}: n \in \omega\right)$ with $G_{0}=H$. 
Proof. Assume that $\mathcal{P}=\mathrm{A}$. By relabeling nodes if necessary, we may assume that $0 \in V\left(G_{0}\right)$. We will show how to construct a chain of graphs $\left(G_{n}: n \in \omega\right)$ with the following property: for each $n \in \omega, n \in V\left(G_{n}\right)$ and $G_{n}$ is a finite induced subgraph of $G$. Note that from this property, it follows that $\lim _{n \rightarrow \infty} G_{n}=G$.

Let $G_{0}=H$. Inductively, assume that $G_{n}$ is defined and finite. For each node $y$ of $G_{n}$, and each subset $X \subseteq N[y]$, let $Y=V\left(G_{n}\right) \backslash X$. Since $G$ has Property (A), there exists a node $z_{X} \in V(G) \backslash V\left(G_{n}\right)$ that is joined to all nodes in $X$, and none in $Y$. Let $V^{\prime}$ be the set of such nodes $z_{X}$, exactly one for each subset $X \subseteq N[y]$ in $G_{n}$. Define $G_{n+1}$ to be the finite subgraph of $G$ induced by $V\left(G_{n}\right) \cup V^{\prime} \cup\{n+1\}$. It is clear that, for all $n \in \omega, G_{n} \leq G_{n+1}$, so $\left(G_{n}: n \in \omega\right)$ is a chain. Adding each node $z_{X}$ in $V^{\prime}$ to $G_{n}$ corresponds to an application of process (P1). If the node $n+1$ is not in $V^{\prime}$, then adding $n+1$ to $G_{n}$ corresponds to one application of process $(\mathrm{P} 2)$.

The proof for Property (B) is similar, and so is omitted.

Corollary 2.5. If $\mathcal{P}$ is either $A$ or $B$, then the following are equivalent.

1. The graph $G$ has property $(\mathcal{P})$.

2. The graph $G$ has a $(\mathcal{P})$-constructing sequence.

To end this section and to aid the reader in what follows, we include tables which compare and contrast some of the known properties of $R$ (the unique isomorphism type of graph with the e.c. property) with graphs satisfying Properties (A) or (B). In the first column is a list of the different adjacency properties we consider. If a graph satisfying the property is unique up to isomorphism, then the name of the unique isomorphism type is written in the second column; otherwise, the number of nonisomorphic graphs with the property is listed. A "Yes" in a column means that a graph with the adjacency property has the property of the header of that column, while a "No" means that the graph does not necessarily have the property. A graph $G$ is inexhaustible if for all $x \in V(G), G-x \cong G$. The graph $G$ is locally $H$ if for all $x \in N(x), G \uparrow N(x) \cong H$. A ray is an infinite path that extends indefinitely in one direction; a double ray is an infinite path that extends indefinitely in two directions. A one-way Hamilton path is a spanning subgraph that is a ray, while a two-way Hamilton path is a spanning subgraph that is a double ray. The second column of the second table only refers to whether the connected components of the graph in question have either a oneor two-way Hamilton path. A graph $G$ is indivisible if whenever the nodes of $G$ are coloured red or blue, there is a monochromatic induced subgraph isomorphic to $G$. 


\begin{tabular}{|c||c|c|c|}
\hline Property & Unique model? & Locally $R$ ? & Inexhaustible? \\
\hline \hline e.c. & Yes, $R$ & Yes & Yes \\
\hline mixed $(A)$ & No, $2^{\aleph_{0}}$ & Yes & Yes \\
\hline pure $(A)$ & Yes, $R_{N}$ & Yes & Yes \\
\hline mixed $(B)$ & No, $2^{\aleph_{0}}$ & No & Yes \\
\hline pure $(B)$ & No, $2^{\aleph_{0}}$ & No & Yes \\
\hline
\end{tabular}

Table I.

\begin{tabular}{|c||c|c|c|}
\hline Property & $\aleph_{0}$-Universal? & Hamilton paths? & Indivisible? \\
\hline \hline e.c. & Yes & Yes & Yes \\
\hline mixed $(A)$ & Yes & Yes & Yes \\
\hline pure $(A)$ & Yes & Yes & Yes \\
\hline mixed $(B)$ & No & Yes & No \\
\hline pure $(B)$ & No & Yes & No \\
\hline
\end{tabular}

Table 2.

The results on whether the limits are unique (up to isomorphism) may be found in Section 3. The results on whether the limits are inexhaustible, have Hamilton paths, or are indivisible, may be found in Section 4.

\section{Many Models}

Our main results concern graphs with Properties (A) and (B). We first show that Properties $(\mathrm{A})$ and $(\mathrm{B})$ are not $\aleph_{0}$-categorical; that is, there are many nonisomorphic graphs that satisfy these properties.

A homomorphism from $G$ to $H$ is a mapping $f: V(G) \rightarrow V(H)$ that preserves edges; more precisely, $x y \in E(G)$ implies that $f(x) f(y) \in E(H)$. We usually write $f: G \rightarrow H$ or just $G \rightarrow H$. If $G \rightarrow H$ and $H \rightarrow G$, then we say that $G$ and $H$ are homomorphically equivalent, and write $G \leftrightarrow H$. See [Hahn and Tardif 97] for more on graph homomorphisms.

Theorem 3.I. Let $H$ be a finite graph. Let $G$ be an infinite pure (B) graph with a pure $(B)$-constructing sequence $\left(G_{n}: n \in \omega\right)$, where $G_{0}=H$. Then $H \leftrightarrow G$.

Proof. As $H \leq G$, we have that $H \rightarrow G$. To show that $G \rightarrow H$, we construct a homomorphism by induction on $n \in \omega$. Let $f_{0}$ be the identity map on $G_{0}=$ 
$H$. Suppose that $f_{n}: G_{n} \rightarrow H$ is a homomorphism extending $f_{0}$. Let $z \in$ $V\left(G_{n+1}\right) \backslash V\left(G_{n}\right)$. Then by the definition of (P1), there exist a node $y \in V\left(G_{n}\right)$ and a subset $X$ of $N(y)$, so that $z$ is only joined to nodes of $X$. So in $G_{n+1}$, $N(z) \subseteq N(y)$. We label this node $y$ as $y_{z}$. Since $f_{n}$ is a homomorphism, $f_{n}\left(y_{z}\right) \neq$ $f_{n}(x)$ for all $x \in N\left(y_{z}\right)$. Hence, we may map $z$ to $f_{n}\left(y_{z}\right)$ and preserve edges. Therefore, the map $f_{n+1}: G_{n+1} \rightarrow H$ defined by

$$
f_{n+1}(z)= \begin{cases}f_{n}(z) & \text { if } z \in V\left(G_{n}\right) \\ f_{n}\left(y_{z}\right) & \text { else }\end{cases}
$$

is a homomorphism. The map $F: G \rightarrow H$ defined by $\bigcup_{n \in \omega} f_{n}$ is a homomorphism.

The following corollary is immediate from Theorem 3.1. The clique number of $G$, written $\omega(G)$, is the maximum cardinality of the vertex set of a complete subgraph in $G$. The chromatic number of $G$, written $\chi(G)$, is the minimum integer $n$ so that we may partition $V(G)$ into $n$ independent sets.

Corollary 3.2. For a fixed finite graph $H$, let $G(H)$ be an infinite pure (B) graph with a pure (B)-constructing sequence $\left(G_{n}: n \in \omega\right)$ such that $G_{0}=H$. Then the following hold:

1. $\chi(G(H))=\chi(H)$ and $\omega(G(H))=\omega(H)$.

2. If $H$ and $H^{\prime}$ are not homomorphically equivalent, then $G(H) \supsetneqq G\left(H^{\prime}\right)$.

We note that there are infinite families of nonhomomorphically equivalent finite graphs; see [Bonato 02]. Hence, there are at least $\aleph_{0}$ many nonisomorphic pure (B) graphs; see Theorem 3.5. This contrasts with the situation for pure (A) graphs.

Theorem 3.3. There is a unique pure (A) graph, up to isomorphism.

We will defer the proof of Theorem 3.3 to Section 4, since our proof will make heavy use of the inexhaustibility property which is discussed there. If $n$ is a positive integer, then a graph $G$ is $n$-existentially closed or $n$-e.c. if each $n$-subset $S$ of $V(G)$ can be extended in all ways. Hence, a graph $G$ is e.c. if it is $n$-e.c. for all positive integers $n$. It is well known that for every constant $p \in(0,1)$, and fixed $n$ a positive integer, almost all finite random graphs with edges chosen independently with probability $p$ are $n$-e.c. We use this property 
to give the maximum possible cardinality of nonisomorphic mixed graphs with Property (A).

Theorem 3.4. There are $2^{\aleph_{0}}$ many nonisomorphic infinite mixed graphs with Prop$\operatorname{erty}(A)$.

Proof. Fix $n \geq 5$. Let $G_{0}=C_{n+1}$, the chordless cycle on $n+1$ nodes. Assume that $G_{i}$ is defined and finite. To form $G_{i+1}$, first apply process (P1) and extend all subsets of closed neighbour sets of $G_{i}$ to form $G_{i+1}^{\prime}$. Then apply process (P2) a finite number of times by extending all $n$-subsets of nodes of $G_{i+1}^{\prime}$ to form $G_{i+1}$. Define $G(n)=\lim _{n \rightarrow \infty} G_{i}$. The graph $G(n)$ has Property (A) with $\left(G_{i}: i \in \omega\right)$ an (A)-constructing sequence, and is clearly $n$-e.c. Note that $G(n)$ is mixed, since (P2) is used in the constructing sequence. However, there is no node in $G(n)$ that is joined to each node of $G_{0}$, so $G(n)$ is not $(n+1)$-e.c. To see this, we proceed by induction on $i$. Assume that there is no node in $G_{i}$ joined to all of $G_{0}$. In $G_{i+1}$, the nodes that are added to $G_{i}$ are of two types: 1 ) extending subsets of closed-neighbour sets in $G_{i}$, or 2 ) extending arbitrary $n$-subsets in $G_{i}$. The nodes of type 2 can never be joined to all the $n+1$ nodes of $G_{0}$. Now consider nodes of type 1. Assume, to obtain a contradiction, that $V\left(G_{0}\right) \subseteq N[y]$ for some $y$ in $G_{i}$. Then $y$ cannot equal an element of $G_{0}$, as $G_{0}$ contains no universal nodes. Hence, $V\left(G_{0}\right) \subseteq N(y)$ which contradicts our induction hypothesis. Therefore, there is no type 1 node in $V\left(G_{i+1}\right) \backslash V\left(G_{i}\right)$ joined to each node of $G_{0}$.

Since any $n$-e.c. graph, where $n \geq 2$, is connected, it follows that each graph $G(n)$ is connected. Now, let $X$ be an infinite subset of $\omega$, listed as $X=\left\{n_{i}: i \in\right.$ $\omega\}$. Define

$$
G(X)=\biguplus_{i \in \omega} G\left(n_{i}\right) .
$$

Hence, the connected components of $G(X)$ are the $G\left(n_{i}\right)$. Then $G(X)$ satisfies (A), since Property (A) is preserved by taking disjoint unions, as is readily verified. Let $Y$ be an infinite subset of $\omega$ with $X \neq Y$. Let $n \in X \backslash Y$. Then $G(X)$ contains a connected component that is $n$-e.c. but not $(n+1)$-e.c. However, there is no such connected component in $G(Y)$; thus, $G(X) \supsetneqq G(Y)$. As there are $2^{\aleph_{0}}$ many infinite subsets of $\omega$, there are $2^{\aleph_{0}}$ many nonisomorphic (A) graphs. As there is a unique isomorphism type of pure (A) graph by Theorem 3.3, there are $2^{\aleph_{0}}$ many nonisomorphic mixed (A) graphs.

Theorem 3.5. There are $2^{\aleph_{0}}$ many nonisomorphic infinite mixed graphs with Property $(B)$ but not $(A)$. There are exactly $\aleph_{0}$ many nonisomorphic infinite pure graphs with Property (B) but not (A). 
Proof. Let $G$ be a pure (B) graph, with a (B)-constructing sequence $\left(G_{i}: i \in \omega\right)$ so that $G_{0}=K_{n}$, for a fixed $n \geq 4$. Since $G$ is pure, $G_{i+1}$ is obtained from $G_{i}$ only by process $(\mathrm{P} 1)$, for each positive integer $i$. By Corollary $3.2, \chi(G)=$ $\chi\left(G\left(K_{n}\right)\right)=n$.

Claim 3.6. $G=G^{\prime} \uplus \overline{K_{\aleph_{0}}}$, where $G^{\prime}$ is a connected graph with $\chi\left(G^{\prime}\right)=n$.

To see this, note that $G_{i+1}$ was constructed by adding nodes joined to some or no nodes of $G_{i}$. Suppose that $G_{i}=G_{1}(i) \uplus G_{2}(i)$, where $G_{1}(i)$ is connected and $G_{2}(i)$ is independent. Let $G_{1}(i+1)$ be those nodes in $G_{i+1}$ that are joined to some node of $G_{i}$, and let $G_{2}(i+1)$ be the nodes in $G_{i+1}$ that are not joined to any node of $G_{i}$. By the definition of (P1), the new nodes of $G_{i+1}$ are either joined to the neighbourhood of a node in $G_{i}$, and thus must be connected to $G_{1}(i)$, or they are independent, and hence, they are part of $G_{2}(i+1)$. Therefore, $\left(G_{1}(i+1)\right)$ is connected and contains $G_{1}(i)$, and $G_{2}(i+1)$ forms an independent set in $G_{i+1}$ and contains $G_{2}(i)$. Moreover, $G_{2}(i+1)$ properly contains $G_{2}(i)$, because according to process (P1), for every node $z$ of $G_{i}$, for the choice $X=\emptyset$, a new independent node $z_{X}$ is added. Note that $G_{i+1}=G_{1}(i+1) \uplus G_{2}(i+1)$. Note also that $G_{0}=K_{n} \uplus H_{0}$, where $H_{0}$ is the empty graph. Let $\lim _{i \rightarrow \infty}\left(G_{1}(i)\right)=G^{\prime}$ and let $\lim _{i \rightarrow \infty}\left(G_{2}(i)\right)=H$. The graph $G^{\prime}$ is connected, as each graph $G_{1}(i)$ is connected, and $H$ is independent, since each $G_{2}(i)$ is independent. Since the cardinality of $G_{2}(i)$ is strictly increasing, $H \cong \overline{K_{\aleph_{0}}}$. Also, since $G(i)=$ $G_{1}(i) \uplus G_{2}(i)$ for each $i$, and $\lim _{i \rightarrow \infty} G_{i}=G, G=G^{\prime} \uplus H$. As $G_{0} \leq G^{\prime} \leq G$, it is immediate that $G^{\prime}$ has chromatic number $n$.

Now let $\Omega=\left\{K_{n}: n \geq 4\right\}$. For a fixed infinite $X \subseteq \Omega$, define a graph $G(X)$ as follows. Let $X=\left\{K_{n_{i}}: i \in \omega\right\}$. As in Corollary 3.2, let $G\left(K_{n_{i}}\right)$ be the graph obtained from $G_{0}=K_{n_{i}}$ by a pure (B)-constructing sequence. By setting $G\left(K_{n_{i}}\right)$ to be the graph $G$ defined earlier in the proof, we obtain that $G\left(K_{n_{i}}\right)=G^{\prime}\left(n_{i}\right) \uplus \overline{K_{\aleph_{0}}}$, where $G^{\prime}\left(n_{i}\right)$ is a connected graph with $\chi\left(G^{\prime}(i)\right)=n_{i}$. Define $G(X)$ as $\biguplus_{i \in \omega} G\left(K_{n_{i}}\right)$. Then $G(X)$ has Property (B), but note that $G(X)$ cannot have Property (A) by Theorem 2.2, since the chromatic number of each connected component is finite. Now if $X, Y$ are infinite subsets of $\Omega$ with $X \neq Y$, then suppose that $K_{n} \in X \backslash Y$. By Claim 3.6, there is no component in $G(Y)$ with chromatic number $n$, so $G(X) \nsucceq G(Y)$. Hence, there are $2^{\aleph_{0}}$ many nonisomorphic (B) graphs.

Let $G$ be a pure (B) graph with pure (B)-constructing sequence $\left(G_{i}: i \in \omega\right)$. It is not hard to see that $G$ is determined up to isomorphism by the finite graph $G_{0}$. As there are only $\aleph_{0}$ many nonisomorphic choices for $G_{0}$, there are at most $\aleph_{0}$ nonisomorphic pure (B) graphs. By Corollary 3.2, there are at least $\aleph_{0}$ nonisomorphic pure (B) graphs, at least one with chromatic number $n$, for each 
$n \in \omega$. Hence, there are exactly $\aleph_{0}$ many nonisomorphic pure (B) graphs, and therefore, by the last sentence in the previous paragraph, there are $2^{\aleph_{0}}$ many nonisomorphic mixed (B) graphs.

We note that the infinite random graph $R$ has Property (A) (and therefore (B)), but has neither a pure (A)- nor (B)-constructing sequence. To see this in the case of Property (A), we note that any pure (A) graph is disconnected. Let $G$ be a pure (A) graph with pure (A)-constructing sequence $\left(G_{i}: i \in \omega\right)$. At each time-step $G_{n}$, where $n>1$, at least two isolated nodes are introduced. For a fixed $n>1$, call two such nodes in $G_{n} u$ and $v$. An inductive argument shows that in the following time-steps $G_{r}$, with $r>n, u$ and $v$ remain in different components of $G_{r}$. Hence, there are at least two connected components in $G$. However, since $R$ is e.c., it is connected of diameter 2. Therefore, $R$ cannot have a pure (A)-constructing sequence. We proved in Corollary 3.2 that any pure (B) graph has finite chromatic number, and so $R$ cannot have a pure (B)-constructing sequence.

We emphasize that it is not claimed here that all of the nonisomorphic (A)or (B)-graphs can occur as limits of the copying model process.

\section{Fractal and Other Properties}

Recall that a graph $G$ is inexhaustible if for all $x \in V(G)$, we have that $G-x \cong G$. The graph $R$ is inexhaustible, as are the infinite complete and null graphs. For more on inexhaustible graphs, the reader is directed to [Bonato and Delić 04] and [Fraïssé 00]. Inexhaustibility is an example of a fractal property of graphs. For more on fractal properties of graphs, the reader is directed to [Bonato et al. 02]. While we do not claim that the inexhaustibility of graphs with Properties (A) or (B) has an explicit relationship to the self-similarity properties observed in the web graph (see [Dill et al. 01]), we think the following result is suggestive.

Theorem 4.I. If $G$ is a fixed graph with Property (B), then $G$ is inexhaustible.

Proof. Let $\left(G_{n}: n \in \omega\right)$ be a (B)-constructing sequence for $G$. If $n$ is a positive integer, then a set of nodes in $G_{n}$ is called $n$-special if it includes nodes of $V\left(G_{n}\right) \backslash V\left(G_{n-1}\right)$. We introduce the following notation for subsets of $V(G)$. Let $S_{1}$ be the set of nodes added to $G_{0}$ in time-step 1 by extending sets of nodes of $G_{0}$. In general, in $G_{r}$, let $S_{r}$ be the set of nodes extending $G_{r-1}$. Let $S_{1,1}=S_{1}$. In $S_{2}$, there are nodes $S_{2,1} \subseteq S_{2}$ extending $G_{0}$ as $S_{1}$ does, and nodes $S_{2,2} \subseteq S_{2}$ extending 1-special sets of nodes. Note that $S_{2}=S_{2,1} \cup S_{2,2}$ and $S_{2,1} \cap S_{2,2}=\emptyset$. 
In general, in $S_{r}$ we define a finite sequence $\left(S_{r, i}: 1 \leq i \leq r\right)$ of sets of nodes partitioning $S_{r}$, with each $S_{r, i}$ consisting of the nodes that extend the $(i-1)$ special sets of $G_{r-1}$. In particular, $S_{r, r}$ is the only set extending $(r-1)$-special sets of nodes. If $1 \leq i \leq r-1$, then the nodes in the set $S_{r, i}$ extend the same subsets that $S_{r-1, i}$ does.

Let $G_{\infty, 0}=G_{0}$. To define $G_{\infty, 1}$, we form disjoint sets of nodes $\left(S_{\infty, 1, i}: i \in \omega\right)$, each disjoint from $V\left(G_{\infty, 0}\right)$ and of the same cardinality as $S_{1,1}$, and let

$$
V\left(G_{\infty, 1}\right)=V\left(G_{\infty, 0}\right) \cup \bigcup_{i \in \omega} S_{\infty, 1, i}
$$

Now let each $S_{\infty, 1, i}$ extend $G_{0}$ as $S_{1,1}$ does. More precisely, the subgraph of $G_{\infty, 1}$ induced by $V\left(G_{0}\right)$ and $S_{\infty, 1, i}$ is isomorphic to $G_{1}$. Moreover, $\bigcup_{i \in \omega} S_{\infty, 1, i}$ is an independent set in $G_{\infty, 1}$. It follows from the definition that $G_{\infty, 1}$ contains infinitely many subgraphs isomorphic to $G_{1}$.

Assume that $G_{\infty, r}$ is defined, countable, and contains infinitely many subgraphs isomorphic to $G_{r}$. To define $G_{\infty, r+1}$, form disjoint sets of nodes $\left(S_{\infty, r, i}\right.$ : $i \geq r)$, each disjoint from $V\left(G_{\infty, 0}\right)$ and of the same cardinality as $S_{r, r}$. Let

$$
V\left(G_{\infty, r}\right)=V\left(G_{\infty, r-1}\right) \cup \bigcup_{i \geq r} S_{\infty, r, i}
$$

Let each of the $S_{\infty, r, i}$ extend one of the subgraphs of $G_{\infty, r}$ isomorphic to $G_{r}$ as $S_{r, r}$ does, and let $\bigcup_{i \geq r} S_{\infty, r, i}$ form an independent set in $G_{\infty, r+1}$. Clearly, $G_{\infty, r+1}$ contains infinitely many subgraphs isomorphic to $G_{r}$. Define $G_{\infty}=$ $\lim _{r \rightarrow \infty} G_{\infty, r}$. See Figure 1.
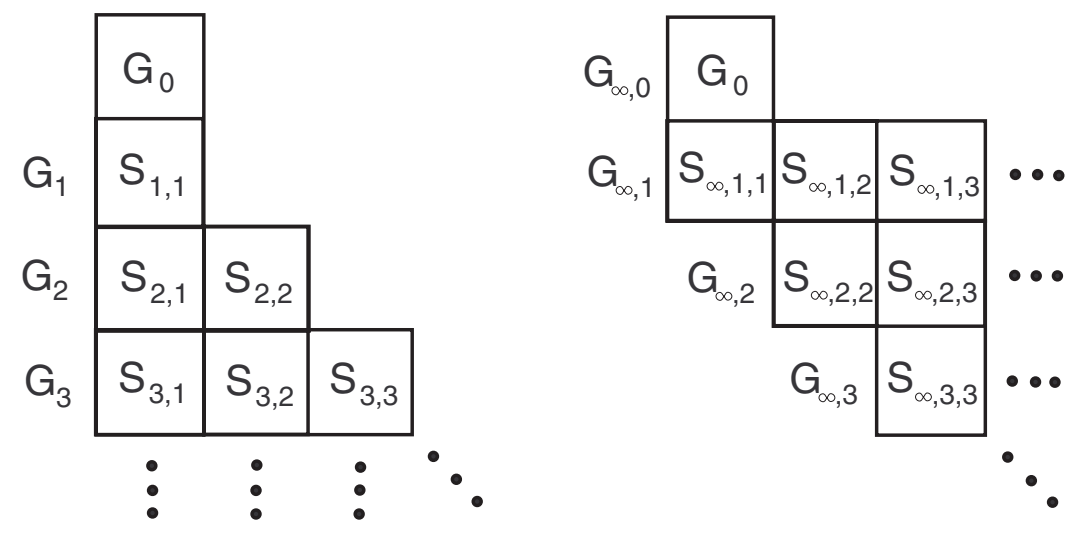

Figure I. The graphs $G$ and $G_{\infty}$. 
Claim 4.2. $G \cong G_{\infty}$.

We define a mapping $f: G \rightarrow G_{\infty}$ as follows. The map $f$ sends $G_{0}$ to $G_{\infty, 0}$ via the identity map. For each $i, j \in \omega \backslash\{0\}$, map $S_{i, j}$ isomorphically onto $S_{\infty, j, i}$. (Hence, "columns" in $G$ are mapped to "rows" in $G_{\infty}$; see Figure 1.) It is straightforward to see that $f$ is an isomorphism. Claim 4.2 follows.

Now, we prove that $G_{\infty}$ is inexhaustible. Fix $x \in V\left(G_{\infty}\right)$.

Case I: $x \notin V\left(G_{\infty, 0}\right)$.

Let $m$ be the largest nonnegative integer so that $G_{\infty, m}$ does not contain $x$. Let $f_{m}: G_{\infty, m} \rightarrow G_{\infty, m}$ be the identity mapping.

Claim 4.3. For all $r>m, G_{\infty, r} \cong G_{\infty, r}-x$ via an isomorphism $f_{r}$ extending $f_{r-1}$.

We proceed by induction on $r$. We use the back-and-forth method to define $f_{r}$, which is a two-player game of perfect information played in countably many steps on two graphs $X_{0}, X_{1}$. The players are named the duplicator and the spoiler. (The names come from the facts that the duplicator is trying to show the graphs are alike, while the spoiler is trying to show they are different.) A move consists of a choice of node from either graph, and the spoiler makes the first move. The players take turns choosing nodes from the $V\left(X_{i}\right)$, so that if one player chooses a node from $V\left(X_{i}\right)$, the other must choose a node of $V\left(X_{i+1}\right)$ (the indices are $\bmod 2$ ). The game begins in our case with a fixed isomorphism $f$ between two induced subgraphs $Y_{0}$ and $Y_{1}$ of $X_{0}$ and $X_{1}$, respectively. Players cannot choose previously chosen nodes, or nodes in a $Y_{i}$. After $n$ rounds, this gives rise to a list of nodes $Y_{0} \cup\left\{a_{i}: 1 \leq i \leq n\right\}$ from $X_{0}$ and $Y_{1} \cup\left\{b_{i}: 1 \leq i \leq n\right\}$ from $X_{1}$. The duplicator wins if for every $n \geq 1$, the subgraph induced by $Y_{0} \cup\left\{a_{i}: 1 \leq i \leq n\right\}$ is isomorphic to the subgraph induced by $Y_{1} \cup\left\{b_{i}: 1 \leq i \leq n\right\}$ via an isomorphism extending $f$ mapping $a_{i}$ to $b_{i}$, for every $1 \leq i \leq n$. Otherwise, the spoiler wins. From this it follows that the duplicator has a winning strategy if and only if $X_{0}$ and $X_{1}$ are isomorphic via an isomorphism extending $f$. See [Cameron 97a] for more on the back-and-forth method.

We let

$$
X_{0}=G_{\infty, r}, X_{1}=G_{\infty, r}-x, f=f_{r-1}, Y_{0}=G_{\infty, r-1}, Y_{1}=G_{\infty, r-1}-x .
$$

Going forward, suppose that the spoiler chooses $y$ in $G_{\infty, r}$, where $y$ is not in $G_{\infty, r-1}$. We will assume that $y$ is a node added at time-step $G_{r}$ by process (P1) (the argument for process (P2) is similar and so is omitted). Then $y$ extends some set $A$ in $N(z)$, for some $A$ and $z$ in $G_{\infty, r-1}$. Let $A^{\prime}=f_{r-1}(A)$, and $z^{\prime}=f_{r-1}(z)$ in $G_{\infty, r-1}-x$. Hence, there is a $y^{\prime}$ in $G_{\infty, r}$ extending $A^{\prime}$ as $y$ extends $A$. If 
$y^{\prime} \neq x$, then the duplicator chooses this node. If $y^{\prime}=x$, then the duplicator may choose any of the infinitely many nodes in $G_{\infty, r}-x$ that also extends $A^{\prime}$ as $y^{\prime}$ does. Going back is similar. Since any two nodes in $V\left(G_{\infty, r}\right) \backslash V\left(G_{\infty, r-1}\right)$ are nonjoined, Claim 4.3 follows.

The map $\bigcup_{r \in \omega} f_{r}: G_{\infty} \rightarrow G_{\infty}-x$ is an isomorphism.

Case 2: $x \in V\left(G_{\infty, 0}\right)$.

Given the finite graph $G_{\infty, 0}$, define the infinite graph $G_{\infty, 0}^{\prime}$ as follows. For each node $y$ of $G_{\infty, 0}$, add infinitely many new pairwise nonjoined nodes $y_{i}$ with the property that $y_{i}$ has the same neighbours in $G_{\infty, 0}$ as $y$ does. (We think of the $y_{i}$ as nodes extending $N(y)$. Hence, $G_{\infty, 0}^{\prime} \leq G_{\infty, 1}$ in $G_{\infty}$.) It is straightforward to see that $G_{\infty, 0}^{\prime}$ is inexhaustible. (See Figure 2 for a depiction of the graph $C_{4}^{\prime}$.)

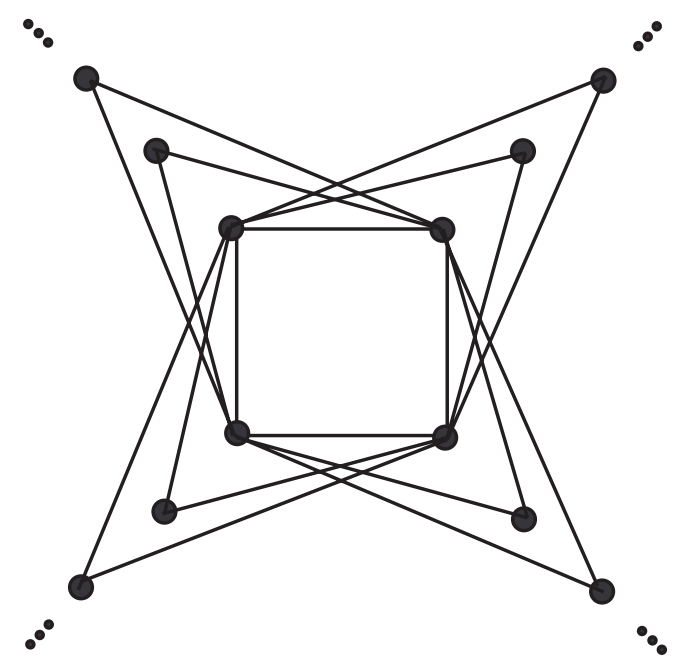

Figure 2. The graph $C_{4}^{\prime}$.

Define a new graph $G_{\infty}^{\prime}$ that is constructed as $G_{\infty}$ was, but beginning with $G_{\infty, 0}^{\prime}$, rather than $G_{\infty, 0}$.

Claim 4.4. $G_{\infty}^{\prime} \cong G_{\infty}$.

To prove Claim 4.4, we first prove that $G_{\infty, 1}^{\prime}$ and $G_{\infty, 1}$ are isomorphic by extending the identity mapping $g_{0}$ between $G_{\infty, 0} \leq G_{\infty, 1}^{\prime}$ and $G_{\infty, 0} \leq G_{\infty, 1}$. Suppose that the spoiler chooses a node $y^{\prime}$ in $V\left(G_{\infty, 1}^{\prime}\right) \backslash V\left(G_{\infty, 0}\right)$. Consider the case when $y^{\prime}$ was added by process (P1). (The argument for the case when $y^{\prime}$ is added by process (P2) is similar, and so is omitted.) The duplicator can respond 
with a node $y \in V\left(G_{\infty, 1}\right)$ joined to $A \subseteq V\left(G_{\infty, 0}\right)$. Going back is similar. Since no two nodes in $V\left(G_{\infty, 1}\right) \backslash V\left(G_{\infty, 0}\right)$ or in $V\left(G_{\infty, 1}^{\prime}\right) \backslash V\left(G_{\infty, 0}\right)$ are joined, as we noted in Case 1, the duplicator can win. Using similar arguments, we obtain, for each $r \in \omega$, isomorphisms $g_{r}: G_{\infty, r}^{\prime} \rightarrow G_{\infty, r}$ so that if $r \geq 1$, then $g_{r}\left\lceil G_{\infty, r-1}^{\prime}=g_{r-1}\right.$. The map

$$
\bigcup_{r \in \omega} g_{r}: G_{\infty}^{\prime} \rightarrow G_{\infty}
$$

is an isomorphism.

To finish Case 2, it is therefore sufficient to show that $G_{\infty}^{\prime}$ is inexhaustible. Choose $y \in V\left(G_{\infty}^{\prime}\right)$. If $y \in V\left(G_{\infty, 0}^{\prime}\right)$, since $G_{\infty, 0}^{\prime}$ is inexhaustible, there is an isomorphism $g_{0}: G_{\infty, 0}^{\prime} \rightarrow G_{\infty, 0}^{\prime}-y$. As in Case 1, extend $g_{0}$ to isomorphisms $g_{r}: G_{\infty, r}^{\prime} \rightarrow G_{\infty, r}^{\prime}-y$, for all $r \geq 0$, by the back-and-forth method. The map

$$
\bigcup_{r \in \omega} g_{r}: G_{\infty}^{\prime} \rightarrow G_{\infty}^{\prime}-x
$$

is an isomorphism. If $y \notin V\left(G_{\infty, 0}^{\prime}\right)$, then proceed as in Case 1 .

Recall that a graph that has an (A)-constructing sequence is pure if at each time-step, process (P1) is used; it is mixed otherwise. One may ask whether there are many nonisomorphic pure (A) graphs. Using Theorem 4.1, we prove that the answer is negative, and thereby prove Theorem 3.3 of Section 3 above. This is in stark contrast to the situation for mixed (A) graphs, as proved in Theorem 3.4. If $G_{0}$ is a finite graph, we use the notation $\uparrow G_{0}$ for the unique (up to isomorphism) graph that results by applying the (P1) process for Property (A) recursively to $G_{0}$. It follows that every pure (A) graph is of the form $\uparrow G_{0}$ for some finite graph $G_{0}$. It is not hard to see that $\uparrow(G \uplus H) \cong \uparrow G \uplus \uparrow H$.

Proof of Theorem 3.3. It is enough to show that if $G_{0}$ and $H_{0}$ are finite graphs, then $\uparrow G_{0} \cong \uparrow H_{0}$. For this let $\left(G_{i}: i \in \omega\right)$ and $\left(H_{i}: i \in \omega\right)$ be (A)-constructing sequences for $G=\uparrow G_{0}$ and $H=\uparrow H_{0}$, respectively. As $H$ is $\aleph_{0}$-universal by Theorem 2.2, $G \leq H$. In particular, there is some $n \in \omega$ so that $G_{0} \leq H_{n}$. Delete from $H$ all the finitely many nodes in $S=V\left(H_{n}\right) \backslash V\left(G_{0}\right)$. At time-step $n+1$, we are left with a copy of $G_{1}$ extending $G_{0}$ (as it is extended in $G$ ), and finitely many isolated nodes, say $m$ of them (that were either joined to no node of $H_{n}$ in $H$, or were joined only to nodes that we deleted). Hence, $H_{n+1}-S \cong G_{1} \uplus \overline{K_{m}}$. Since $G_{1}$ extends $G_{0}$, we have that $H-S \cong \uparrow\left(G_{0} \uplus \overline{K_{m}}\right) \cong \uparrow G_{0} \uplus \uparrow \overline{K_{m}}$.

At each time-step $r$ in the construction of $G(r>0)$, nodes with no edges to $G_{r-1}$ are added to $G_{r}$. These nodes give rise to connected components of $G$ of the form $\uparrow K_{1}$. Hence, $G$ contains infinitely many connected components of the form $\uparrow K_{1}$. It follows that $G$ is isomorphic to the graph $J$, which consists of the disjoint union of $\uparrow G_{0}$ and infinitely many disjoint copies of $\uparrow K_{1}$. 
As $H$ is inexhaustible by Theorem 4.1, it follows that $H-S \cong H$. But then

$$
H \cong H-S \cong \uparrow\left(G_{0} \uplus \overline{K_{m}}\right) \cong \uparrow G_{0} \uplus \uparrow \overline{K_{m}} \cong J \cong G,
$$

since $\uparrow \overline{K_{\aleph_{0}}} \uplus \uparrow \overline{K_{m}} \cong \uparrow \overline{K_{\aleph_{0}}}$.

The unique isomorphism type of Theorem 3.3 we name $R_{N}$, since it is locally isomorphic to $R$. We do not know much about $R_{N}$. The infinite random graph $R$ is indivisible; recall that a graph $G$ is indivisible whenever the nodes of $G$ are coloured red or blue, there is a monochromatic induced subgraph isomorphic to G. For more on indivisible graphs, see [El-Zahar and Sauer 89, Fraïssé 00]. A graph without this property is divisible. A graph $G$ so that $R \leq G$ is necessarily indivisible, since $R$ is itself indivisible. Therefore, by Theorem 2.2, a graph with Property (A), such as $R_{N}$, is indivisible. It is not hard to see that a graph with at least one edge and with finite chromatic number is divisible, so by the proof of Theorem 3.4 for (B), there are examples of graphs with Property (B) that are divisible.

To remind the reader of terminology defined earlier, a ray is an infinite path that extends indefinitely in one direction; a double ray is an infinite path that extends indefinitely in two directions. A one-way Hamilton path is a spanning subgraph that is a ray, while a two-way Hamilton path is a spanning subgraph that is a double ray. The graph $R$ contains one- and two-way Hamilton paths.

Theorem 4.5. If $G$ has Property (B), then the connected components of $G$ have oneand two-way Hamilton paths. In particular, $G$ has a 1-factor.

While Theorem 4.5 is not suggestive of properties of the finite graphs resulting from the copying model, it does parallel the known fact that $R$ has one- and twoway Hamilton paths; see [Cameron 97b].

Proof of Theorem 4.5. Let $G^{\prime}$ be a fixed connected component of $G$. We prove that $G^{\prime}$ has a one-way Hamilton path; the existence of a two-way Hamilton path is similar. Without loss of generality, let $V\left(G^{\prime}\right)=\omega$.

Define $P_{0}$ to be the subgraph induced by $\{0\}$. Assume that there is a path $P(n)$ in $G^{\prime}$ containing the nodes $\{0, \ldots, n\}$, and that the nodes of $P(n)$ are $x_{1}, \ldots, x_{s}$. If the node $n+1$ equals some $x_{i}$, then let $P(n+1)=P(n)$. Otherwise, assume that $n+1$ is not a node in $P(n)$. As $G^{\prime}$ is connected, the node $n+1$ is connected by a path $Q$ to some node $x_{i}$ of $P(n)$. Let $Q$ be the path $y_{1} y_{2} \cdots y_{t-1} y_{t}$, where $y_{1}=n+1$ and $y_{t}=x_{i}$. As $x_{i+1}$ and $y_{t-1}$ are in $N\left(x_{i}\right)$, by Property (B) there is a node $z_{1}$ in $G$, and hence, in $G^{\prime}$, that is joined to both $x_{i+1}$ and $y_{t-1}$, and is not a node of $P(n)$ nor $Q$. 


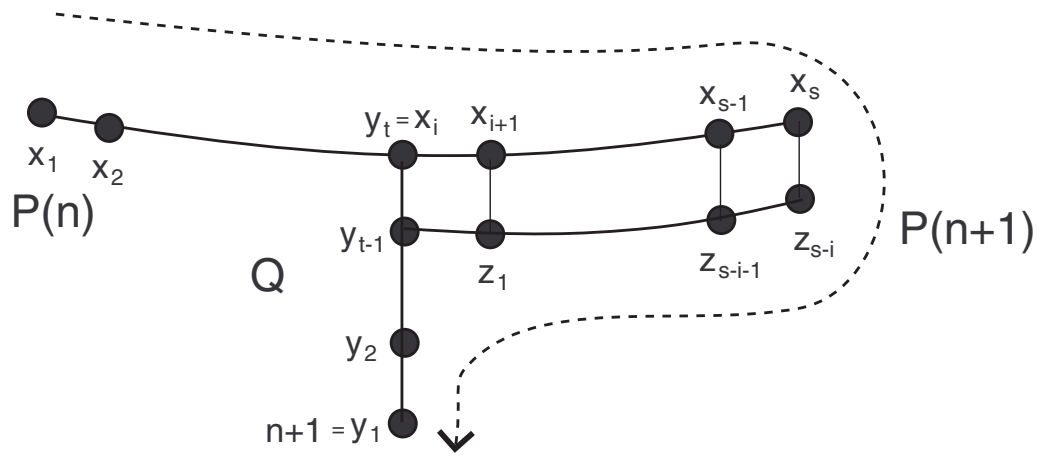

Figure 3. A Hamilton path containing $\{0, \ldots, n+1\}$.

Proceeding inductively, we obtain a path of nodes $z_{1} z_{2} \cdots z_{s-i}$ in $G^{\prime}$ so that $z_{j+1}$ is joined to both $z_{j}$ and $x_{i+j+1}$. Then the path $P(n)$ followed by the path $z_{s-i} \cdots z_{2} z_{1}$ is a path $P(n+1)$ containing $\{0, \ldots, n+1\}$. The desired Hamilton path of $G^{\prime}$ is $\lim _{n \rightarrow \infty} P(n)$. See Figure 3 .

The infinite random graph $R$ contains every countable bipartite graph as an induced subgraph, so there are many such bipartite cores in graphs with the e.c. property, or with Property (A) by Theorem 2.2. The following theorem proves a similar result for graphs with Property (B). The infinite bipartite graph with each vertex class infinite is written $K_{\aleph_{0}, \aleph_{0}}$.

Theorem 4.6. If $G$ is a graph with Property (B) so that $G_{0}$ has at least one edge, then $K_{\aleph_{0}, \aleph_{0}} \leq G$. In particular, there are infinitely many node-disjoint bipartite cores in a graph satisfying Property (B).

Proof. Let $S_{1}$ be some edge of $G$ (by the fact that $G_{0}$ has edges, we know that $G$ also has edges). Assume that $S_{n} \leq G$ is isomorphic to $K_{n, n}$ with $S_{1} \leq S_{n}$. Suppose that $S_{n}$ has nodes $A \cup B$, with $A=\left\{x_{i}: 1 \leq i \leq n\right\}, B=\left\{y_{i}: 1 \leq i \leq\right.$ $n$ \} consisting of independent sets. By Property (B), there is a node $x_{n+1}$ not in $A \cup B$ that is joined to each node of $B$. Since $A \cup\left\{x_{n+1}\right\} \subseteq N\left(y_{1}\right)$, by (B), there is a node $y_{n+1}$ not in $A \cup B \cup\left\{x_{n+1}\right\}$ that is joined to each node of $A \cup\left\{x_{n+1}\right\}$. Then the graph $S_{n+1}$ consisting of $S_{n}$ along with $x_{n+1}$ and $y_{n+1}$ is a bipartite clique strictly containing $S_{n}$. The graph $H=\bigcup_{n>1} S_{n} \leq G$ is isomorphic to $K_{\aleph_{0}, \aleph_{0}}$. 


\section{Acknowledgments}

The authors wish to thank the anonymous referees for numerous excellent suggestions. The authors would like to thank Dejan Delić and Peter Cameron for useful discussions. The authors gratefully acknowledge support from NSERC research grants, and from a MITACS grant. The first author acknowledges support from a Wilfrid Laurier University Senior Research Fellowship.

\section{References}

[Adler and Mitzenmacher 01] M. Adler and M. Mitzenmacher. "Towards Compressing Web Graphs." In Proceedings of the IEEE Data Compression Conference (DCC), pp. 203-212, Los Alamitos, CA: IEEE Press, 2001.

[Aiello et al. 00] W. Aiello, F. Chung, and L. Lu. "A Random Graph Model for Power Law Graphs." In Proc. ACM Symp. on Theory of Computing, pp. 171-180, New York: ACM Press, 2000.

[Aiello et al. 01] W. Aiello, F. Chung, and L. Lu. "Random Evolution in Massive Graphs." In Proceedings of the 42nd IEEE Symposium on Foundations of Computer Science, pp. 510-519, Los Alamitos, CA: IEEE Computer Soc., 2001.

[Barabàsi and Albert 99] A. Barabási and R. Albert. "Emergence of Scaling in Random Networks." Science 286 (1999), 509-512.

[Barabàsi et al. 01] A. Barabási, R. Erzsébet, and T. Vicsek. "Deterministic Scale-Free Networks." Physica A 299 (2001), 559-564.

[Bollobás et al. 01] B. Bollobás, O. Riordan, J. Spencer, and G. Tusnády. "The Degree Sequence of a Scale-Free Random Graph Process." Random Structures Algorithms 18:3 (2001), 279-290.

[Bonato 02] A. Bonato. "A Family of Universal Pseudo-Homogeneous G-Colourable Graphs." Discrete Math. 247:1-3 (2002), 13-23.

[Bonato and Delić 04] A. Bonato and D. Delić. "On a Problem of Cameron's on Inexhaustible Graphs." To appear in Combinatorica.

[Bonato et al. 02] A. Bonato, P. Cameron, D. Delić, and S. Thomassé. "Generalized Pigeonhole Properties of Graphs and Oriented Graphs." Europ. J. Combin. 23:3 (2002), 257-274.

[Cameron 97a] P. J. Cameron. "Oligomorphic Groups and Homogeneous Graphs." In Graph Symmetry, pp. 23-74, NATO Adv. Sci. Inst. Ser. C Math. Phys. Sci., 497. Dordrecht: Kluwer Acad. Publ., 1997.

[Cameron 97b] P. J. Cameron. "The Random Graph." In Algorithms and Combinatorics 14, edited by R. L. Graham and J. Nešetřil, pp. 333-351. New York: Springer Verlag, 1997.

[Cooper and Frieze 01] C. Cooper and A. Frieze. "On a General Model of Web Graphs." In Proceedings of ESA, pp. 500-511, Heidelberg: Springer-Verlag, 2001. 
[Dill et al. 01] S. Dill, R. Kumar, K. McCurley, P. Raghavan, S. Rajagopalan, D. Sivakumar, and A. Tomkins. "Self-Similarity in the Web." In Proceedings of International Conference on Very Large Data Bases, pp. 69-78, New York: ACM Press, 2001.

[El-Zahar and Sauer 89] M. El-Zahar and N. Sauer. "The Indivisibility of the Homogeneous $K_{n}$-Free Graphs." J. Combin. Theory Ser. B 47:2 (1989), 162-170.

[Erdős and Rényi 63] P. Erdős and A. Rényi. "Asymmetric Graphs." Acta Math. Acad. Sci. Hungar. 14 (1963), 295-315.

[Erdős and Rényi 60] P. Erdős and A. Rényi. "On the Evolution of Random Graphs." Publ. Math. Inst. Hungar. Acad. Sci. 5 (1960), 17-61.

[Fraïssé 00] R. Fraïssé. Theory of Relations, Revised edition, with an appendix by Norbert Sauer. Amsterdam: North Holland, 2000.

[Fraïssé 53] R. Fraïssé. "Sur certains relations qui généralisent l'ordre des nombres rationnels." C.R. Acad. Sci. Paris 237 (1953), 540-542.

[Hahn and Tardif 97] G. Hahn and C. Tardif. "Graph Homomorphisms: Structure and Symmetry." In Graph Symmetry, pp. 107-166, NATO Adv. Sci. Inst. Ser. C Math. Phys. Sci. 497. Dordrecht: Kluwer Acad. Publ., 1997.

[Kumar et al. 99] R. Kumar, P. Raghavan, S. Rajagopalan, and A. Tomkins. "Trawling Emerging Cyber-Communities Automatically." In Proc. 8th WWW Conf., pp. 1481-1493, New York: Elsevier North Holland, Inc., 1999.

[Kumar et al. 00a] R. Kumar, P. Raghavan, S. Rajagopalan, D. Sivakumar, A. Tomkins, and E. Upfal. "Stochastic Models for the Web Graph." In Proceedings of the 41th IEEE Symp. on Foundations of Computer Science, pp. 57-65, Los Alamitos, CA: IEEE Press, 2000.

[Kumar et al. 00b] R. Kumar, P. Raghavan, S. Rajagopalan, D. Sivakumar, A. Tomkins, and E. Upfal. "The Web as a Graph." In Proc. 19th ACM SIGACTSIGMOD-AIGART Symp. Principles of Database Systems, pp. 1-10. New York: ACM Press, 2000.

[Lachlan and Woodrow 80] A. H. Lachlan and R. E. Woodrow. "Countable Ultrahomogeneous Undirected Graphs." Trans. Amer. Math. Soc. 262:1 (1980) 51-94.

Anthony Bonato, Department of Mathematics, Wilfrid Laurier University, Waterloo, ON, Canada, N2L 3C5 (abonato@rogers.com)

Jeannette Janssen, Department of Mathematics and Statistics, Dalhousie University, Halifax, NS, Canada, B3H 3J5 (janssen@mathstat.dal.ca)

Received April 21, 2003; accepted August 7, 2003. 
